\title{
Unmet expectations delay sensory processes
}

\author{
Buse M. Urgen ${ }^{\text {a,b, * }}$, Huseyin Boyaci ${ }^{\text {a,b,c,d }}$ \\ a Interdisciplinary Neuroscience Program, Bilkent University, Ankara, 06800, Turkey \\ ${ }^{\mathrm{b}}$ Aysel Sabuncu Brain Research Center \& National Magnetic Resonance Research Center (UMRAM), Bilkent University, Ankara, 06800, Turkey \\ ${ }^{\mathrm{c}}$ Department of Psychology, Bilkent University, Ankara, 06800, Turkey \\ ${ }^{\mathrm{d}}$ Department of Psychology, Justus Liebig University Giessen, Giessen, Germany
}

\section{A R T I C L E I N F O}

\section{Keywords:}

Expectation

Prediction

Visual perception

Perceptual inference

Bayesian theorem

Predictive processing

\begin{abstract}
A B S T R A C T
Expectations strongly affect and shape our perceptual decision-making processes. Specifically, valid expectations speed up perceptual decisions, and determine what we see in a noisy stimulus. Despite the well-established effects of expectations on decision-making, whether and how they affect low-level sensory processes remain elusive. To address this problem, we investigated the effect of expectation on temporal thresholds in an individuation task (detection of the position of an intact image, a house or face). We found that compared to a neutral baseline, thresholds increase when the intact images are of the unexpected category, but remain unchanged when they are of the expected category. Using a recursive Bayesian model with dynamic priors we show that delay in sensory processes is the result of further processing, consequently longer time, required in case of violated expectations. Expectations, however, do not alter internal parameters of the system. These results reveal that sensory processes are delayed when expectations are not met, and a simple parsimonious computational model can successfully explain this effect.
\end{abstract}

\section{Introduction}

Visual input received by the eye is often noisy, weak, incomplete and almost always ambiguous. Thus, the sensory input alone cannot with certainty produce a unique percept. The visual system, however, usually comes up with a unique interpretation of the input. It is widely believed that the visual system achieves this feat by using its prior knowledge about the regularities in the world. This prevalent idea is known as perceptual inference (Helmholtz, 1866). Prior knowledge, and our expectations based on that knowledge, can govern what we see, help us predict future events, and guide our behavior (de Lange, Heilbron, \& Kok, 2018). For example, while searching for a painting, we look at locations where the painting is more likely to be found, e.g. the wall, instead of searching every single item or place in the room. Indeed under laboratory conditions, it has been consistently shown that prior knowledge and expectations influence perceptual decisions (e.g., Bar, 2004, Kok, Jehee, \& De Lange, 2012, Summerfield and De Lange, 2014). More specifically, expected stimulus (the congruent stimulus in a cuedparadigm) is recognized or found faster and more accurately than the unexpected (incongruent) one. The reaction time and accuracy measures used in perceptual decision studies, however, reflect the combined effects of cognitive, motor, and sensory processes. Therefore it is not easy to isolate the effects of expectation on sensory processes using these paradigms. Indeed, whether and how expectations affect early sensory processes is still a matter of intense debate (Rungratsameetaweemana, Itthipuripat, Salazar, \& Serences, 2018; Aitken, Turner, \& Kok, 2020). For instance, do the sensory processes get faster if a stimulus is of an expected category, or do they get delayed if it is of an unexpected category? Do the internal parameters of the system, such as response profiles of neuronal populations, change?

Here we tackle these questions by first behaviorally measuring the effect of category expectation on a detection task. Next, we implement a recursive Bayesian model to explain the behavioral findings, and test whether the internal parameters of the system change with expectation. Our findings expand on the behavioral effects of expectation on low level visual processing by unraveling the computational mechanisms that underlie the perceptual effects we found. We also discuss our findings within the framework of mechanistic neuronal models, including predictive processing models.

\footnotetext{
* Corresponding author at: Interdisciplinary Neuroscience Program, Bilkent University, Ankara 06800, Turkey.

E-mail address: buse.urgen@bilkent.edu.tr (B.M. Urgen).
} 


\section{Materials and methods}

\subsection{Behavioral experiment}

\subsubsection{Participants}

Eight naive participants ( 4 female; $24.5 \pm 2.33$ years) participated in the behavioral experiment, which included four experimental conditions blocked in different experimental runs. All participants had normal or corrected to normal vision and reported no history of neurological disorders. Participants gave their written informed consent prior to the experiment. The experiment was approved by the Research Ethics Committee at Bilkent University.

\subsubsection{Stimuli}

Stimuli consisted of two category of images: ten face images (five female; age range was 19-69) taken from Face Database of the Park Aging Mind Laboratory (Minear \& Park, 2004) and ten house images from Scene Understanding Database from the Princeton Vision Group (Xiao, Hays, Ehinger, Oliva, \& Torralba, 2010). Cues (informative: face, house; uninformative (neutral): question mark) were taken from The Noun Project's website (www.thenounproject.com; House by OCHA Visual Information Unit, Person by Alex Fuller, question mark by Vicons Design from the Noun Project) and were scaled to $3.5 \times 3.5^{\circ}$ visual angle. As mask, scrambled versions of the images were generated by dividing the image into 49 cells via creating $7 \times 7$ grids for each. After that each cell was randomly assigned to different locations. The stimuli including intact images (target stimuli) and mask images were scaled to $10.5 \times 10.5^{\circ}$ visual angle, converted to grayscale, histogram-matched (scaled to the mean luminance of all stimuli) by using SHINE Toolbox (Willenbockel et al., 2010), and adjusted and matched to a very low contrast value (2\%). Experiments were programmed in MATLAB 2016a using Psychtoolbox (Brainard, 1997). Stimuli were shown on a CRT monitor (HP P1230, 22 inches, $1024 \times 768$ resolution, refresh rate 120 Hz.)

\subsubsection{Experimental design}

Stimuli were presented on a gray background (RGB: 128, 128, 128). Each trial started with a cue simultaneously with a fixation dot located on the center of the cue, and presented for $2 \mathrm{~s}$ at the center of the screen. Cues were either informative (face and house) or neutral (question mark) depending on the experimental condition (See Experimental Session for details). Next, a target stimulus, which was an intact face or house image, and a scrambled version of the same image were simultaneously shown in left and right side of the cue at $10^{\circ}$ eccentricity. Presentation duration of these images were determined by an adaptive staircase procedure (See Procedure for details). Next, as masks, different scrambled versions of that target stimulus were shown on the same locations for 64 ms. Following this, an empty display with a gray background was presented until a response is given by the participants. Participants' task was to detect the location of the intact image as soon as possible by pressing the left or right arrow key of the keyboard while maintaining their fixation on the fixation dot during the trial. Finally, a feedback message was given as "correct" or "wrong" for 752 ms. Note that cues were informative about the upcoming target image category while task of the participants was to report the spatial location of the target image, irrespective of its category. Therefore, the expected feature of the target image, i.e. image category, was task-irrelevant to isolate expectation from top-down attention processes. Consequently, there were two trial types: when the category of the cue and the image are the same, these trials are called congruent trials (expected stimulus category). When the category of the cue and the image are different, these trials are called incongruent trials (unexpected stimulus category). Note that equal number of each cue (face and house) appeared in random order in the experimental conditions where an informative cue is presented. Also note that equal number of each target stimulus (face and house image) was presented in all experimental conditions, and the target stimulus was randomly assigned to one of the two locations (left or right) in each trial. See Fig. 1 for sample trials from the experiment.

\subsubsection{Procedure}

Behavioral experiment consisted of a training session and an experimental session that comprises four experimental conditions. In both sessions, 2-down 1-up adaptive staircase procedure with a two alternative forced-choice (2-AFC) paradigm was applied to derive duration thresholds $(70.7 \%$ accuracy) in different trial types: neutral trials, congruent trials and incongruent trials in different conditions (see Experimental Session for details). Presentation duration of the target image and scrambled version of it were varied adaptively from trial to trial. The duration of each trial was determined by the accuracy of the participants' responses in previous trials. Specifically, each wrong answer or two consecutive correct answers resulted in approximately $8 \mathrm{~ms}$ (step size) increase or decrease of the duration of the following trial target presentation respectively. At the beginning of each experimental condition, one staircase started from a relatively short duration (varied for each participant, minimum $8 \mathrm{~ms}$ ), and the other staircase started from a relatively long duration (varied for each participant). There were 30 trials in each staircase in all experimental conditions, but number of staircases varied for each experimental condition.

Training Session. Prior to the experimental session, each participant completed a training session in order to stabilize their perceptual thresholds. Participants were seated $60 \mathrm{~cm}$ away from the screen and their heads were stabilized with a chin-rest. The training session consisted of 2 to 5 short experimental runs where the cue was always informative (face and house cue) and $100 \%$ valid in indicating the target stimulus category. Each run in the training phase had 120 trials and there were equal number of face and house trials. Number of experiments completed in the training phase varied for each participant, and it is determined by whether the participant's threshold stayed within an interval of $8 \mathrm{~ms}$ (step size) for at least two consecutive experimental runs.

Experimental Session. All participants completed four experimental conditions in separate runs in a randomized order. Participants were informed about the cue-validity prior to the experiments. Cue validity refers to the relative frequency that the cue correctly predicts the category of the upcoming intact image.

$100 \%$-validity condition. In this experimental condition the cue (face or house) informed participants about the upcoming target stimulus category (either face or house image) with a $100 \%$ validity so that there was no violation of expectations. There were 120 (congruent) trials in total including 60 trials where the target was a face image following the face-cue, and 60 trials where the target was a house image following the house-cue.

75\%-validity condition. In this experimental condition the cue informed about the correct category of the intact image with $75 \%$ probability (face or house). Equal number of each cue (face and house) were presented, and there were 480 trials in total. There were 360 congruent trials where the image category was correctly predicted by the cue, and 120 incongruent trials where the cue misled the participants about the upcoming image category.

$\mathbf{5 0 \%}$-validity condition. In this experimental condition the cue validity was at $50 \%$. Therefore, in total there were 240 trials, of which 120 were congruent and 120 were incongruent. Equal number of each cue was presented.

Neutral (no expectation) condition. This experimental condition was included as a control condition because there was no informative cue (face or house) that informs participants about the upcoming image category. Rather, the cue was neutral, a question mark. Therefore, expectations about the upcoming stimuli were not formed. Except the cue type, all experimental stimuli and design were the same as in other conditions. There were 120 trials in total, and equal number of each image category was presented. 


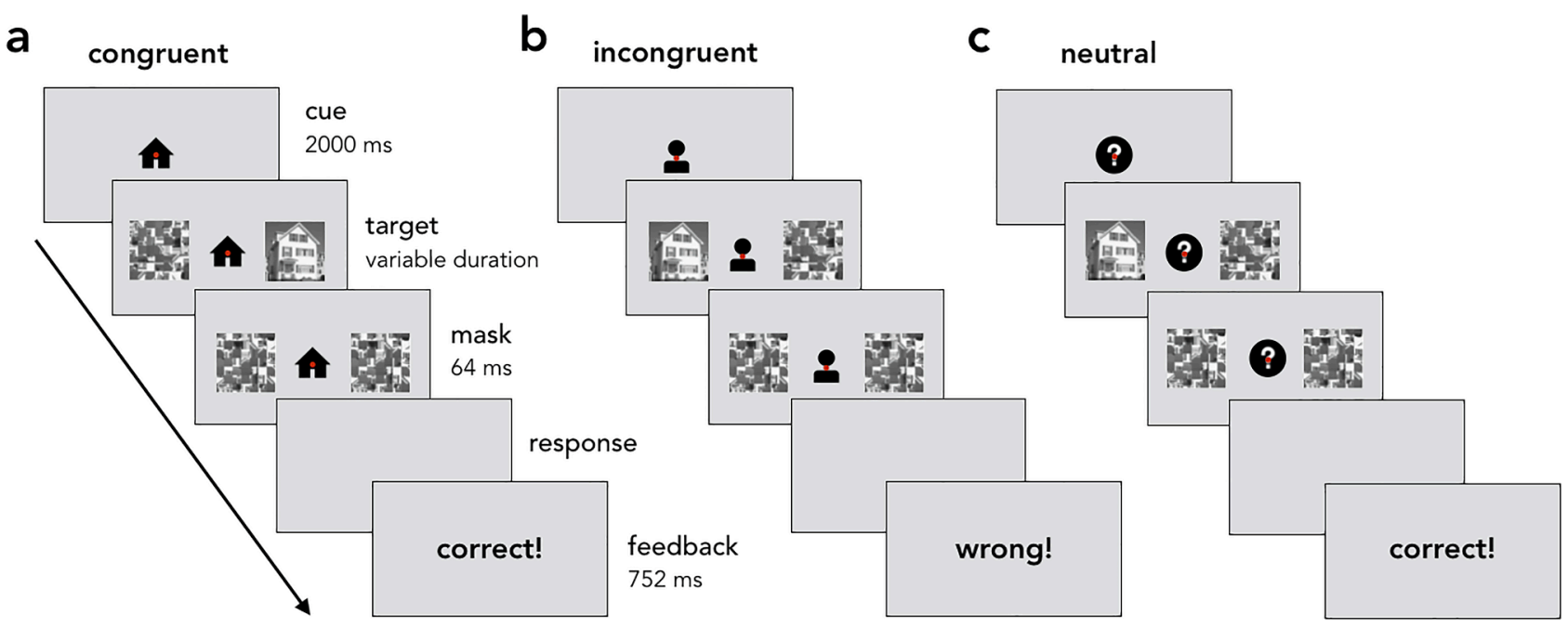

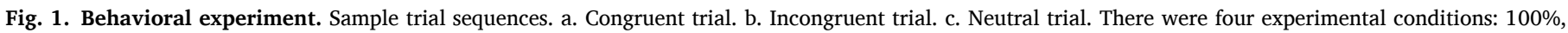

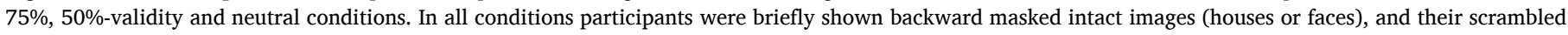

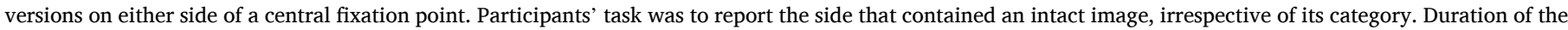

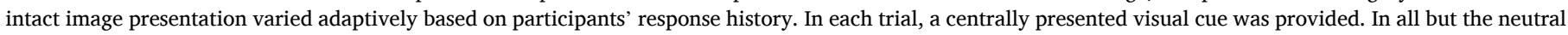

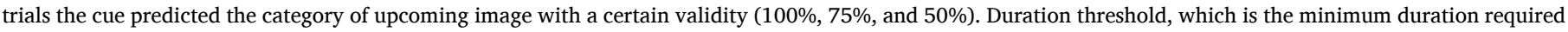

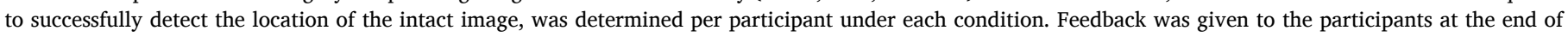
each trial.

\subsubsection{Statistical analysis}

Duration thresholds $(70.7 \%$ accuracy) for location detection in congruent, incongruent and neutral trials were estimated with Logistic function fits using the Palamedes toolbox (Kingdom \& Prins, 2010) routines in Matlab 2016a. A 2 (trial type: congruent, incongruent trial) $\times 2$ (validity: $75 \%, 50 \%$ ) repeated measures ANOVA was conducted to investigate the effect of expectation on duration thresholds. Also, we conducted two-sample two-tailed paired $t$-test to compare the thresholds between the $100 \%$-validity condition and the neutral (no-expectation) condition.

\subsection{Control experiment}

To address the possibility of any confounding effect of training on our behavioral findings, we conducted the experiment without a training session on a separate group of participants.

\subsubsection{Participants}

Ten participants (Group 1: 6 participants, $26 \pm 1.78$ years; Group 2: 4 participants, $26.25 \pm 2.22$ years) participated in the control experiment. All participants had normal or corrected to normal vision and reported no history of neurological disorder. Informed consent was taken prior to the experiment. They were randomly assigned to one of the two experimental groups (See Procedure below for details). Experimental procedures were approved by the Research Ethics Committee at Bilkent University.

\subsubsection{Stimuli, experimental design and procedure}

All stimuli and experimental design were exactly the same as in the main experiment. Participants were randomly assigned to one of the two experimental groups: Group 1 completed two experimental conditions that comprised $75 \%$-validity and neutral conditions, and Group 2 completed 50\%-validity and neutral conditions. Participants completed each experimental condition in random order in separate sessions.

\subsubsection{Statistical analysis}

As in the main experiment duration thresholds for location detection in congruent, incongruent and neutral trials were estimated with Logistic function fits using the Palamedes toolbox (Kingdom \& Prins, 2010) routines in Matlab 2016a. We conducted two-sample two-tailed paired $t$ tests separately for Group 1 and Group 2 to compare the thresholds between the the neutral (no-expectation) condition and 75\%-validity (Group 1) or 50\%-validity (Group 2) condition.

\section{Results}

\subsection{Behavioral results}

In order to investigate the effects of expectation on sensory processes, we measured temporal detection thresholds in an individuation task (Fig. 1). For each participant we computed the duration thresholds (shortest presentation duration for $70.7 \%$ success rate) in congruent (expected stimulus category), incongruent (unexpected stimulus category) and neutral trials. We performed a 2 (trial type: congruent, incongruent trial) $\times 2$ (validity: $75 \%, 50 \%$ ) repeated measures ANOVA to investigate the effect of expectation on duration thresholds. Fig. 2 shows the thresholds averaged across participants as well as individual participant results under each validity condition (see Supplementary Material for psychometric function fits for each participant). We found that the main effect of trial type is statistically significant $(F(1,7)=$ $\left.11.956, p=0.011, \mathrm{n}^{2}=0.104\right)$. However, the main effect of validity and interaction were not significant $\left(F(1,7)=2.848, p=0.135, \mathrm{n}^{2}=0.024 ; F\right.$ $\left.(1,7)=0.155, p=0.705, \mathrm{n}^{2}=0.001\right)$. Next, we examined whether the thresholds of congruent and incongruent trials differ in each validity condition. As expected, our results showed that the difference between the thresholds was significant in the $75 \%$-validity condition $(t(7)=$ 3.079, $p=0.018, d=1.089$ ), indicating that unexpected stimuli led to significantly longer duration thresholds than expected stimuli. However, there was no significant difference in the $50 \%$-validity condition $(t(7)=$ $2.266, p=0.058, d=0.801$ ). Finally, we conducted two-sample twotailed paired t-tests between (1) the $100 \%$-validity and neutral conditions, (2) the congruent trials of $75 \%$ - and $100 \%$-validity conditions, and (3) the congruent trials of $50 \%$ - and $100 \%$-validity conditions. Surprisingly, however, none of those tests yielded significant results $(t(7)=$ $0.676, p=0.521, d=0.239 ; t(7)=0.457, p=0.661, d=0.162 ; t(7)=$ 1.404, $p=0.203, d=0.496)$.

Next, we examined the possibility of any confounding effect of training on our behavioral findings (recall that in the main experiment 

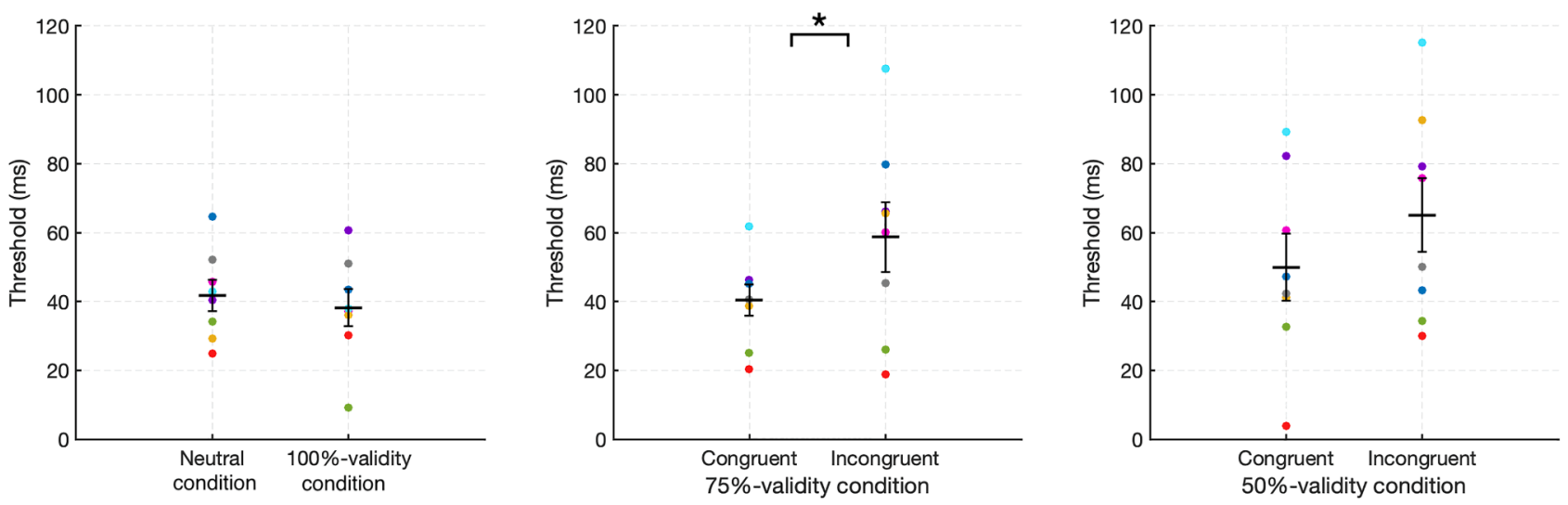

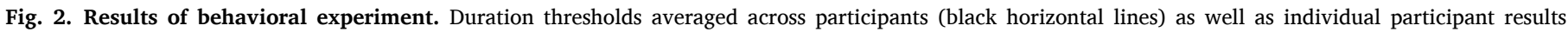

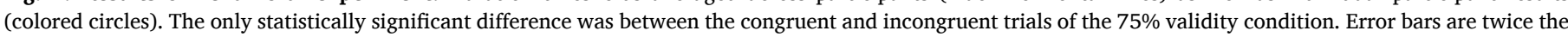
standard error.

participants completed a training session before the experimental session, where only $100 \%$ validity trials were used). For this aim, we conducted a control experiment on a separate group of participants who did not participate in a training session prior to the experimental sessions: Group 1 participated in $75 \%$-validity and neutral conditions, and Group 2 participated in 50\%-validity and neutral conditions. Results of the control experiment confirmed the findings of the main experiment (see Fig. 3). In 75\%-validity condition there was statistically significant difference between congruent and incongruent trials $(t(5)=2.782$, $p=0.039, d=1.136)$. In $50 \%$-validity condition there was not a significant difference between congruent and incongruent trials $(t(5)=$ $0.071, p=0.948, d=0.036)$. Also, thresholds of neutral condition were not different from congruent trials of 75\%-(Group 1) $(t(5)=0.148$, $p=0.888, d=0.061$ ) and 50\%- validity (Group 2 ) conditions $(t(5)=$ $-0.724, p=0.521, d=0.362$ ). In short, results of the control experiment confirmed that the findings of the main experiment are not due to an effect of training.

Taken together, these results show that valid expectations do not reduce the thresholds. Rather, the sensory thresholds increase when the expectations are high but they are not met. There are two alternatives that may explain this finding. First, it is possible that the underlying parameters of the system (e.g. neuronal response profiles or decision criteria) may be altered based on expectation and/or its validity, such that completion of the sensory process requires longer time in an incongruent trial when expectations are high (De Loof, Van Opstal, \& Verguts, 2016; Bang \& Rahnev, 2017). Alternatively, it is possible that in incongruent trials simply further processing may be required to

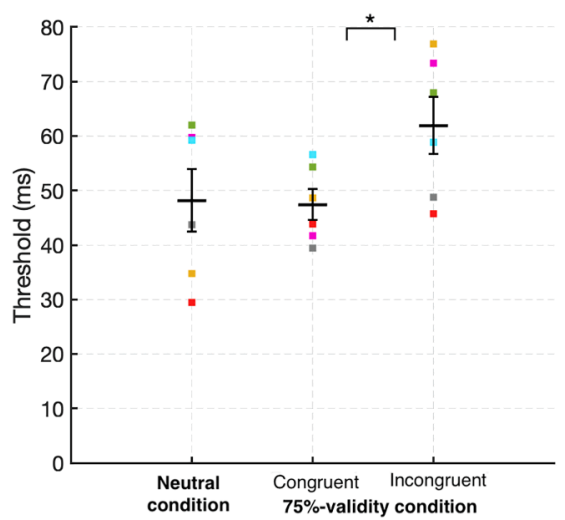

converge on a decision because expectation and the actual input disagree. The standard psychophysical analysis alone cannot inform as to which of these alternatives better explains the behavioral results. Thus, in order to pit the two alternatives against each other, we modeled the behavioral data using the Bayesian theorem, explained next.

\subsection{Bayesian model}

Bayesian theorem provides a compelling mathematical framework to formalize inferential processes in the visual system (Knill \& Richards, 1996; Knill \& Pouget, 2004). Within this framework, the system combines the information about the incoming sensory input with its own priors to infer the most probable causes of that sensory input (Mamassian, Landy, \& Maloney, 2002; Weiss, Simoncelli, \& Adelson, 2002; Kersten \& Yuille, 2003; Kersten, Mamassian, \& Yuille, 2004; Yuille \& Kersten, 2006; Maloney \& Mamassian, 2009). Bayesian theorem has also been successful to formally describe how prior knowledge and expectations shape perceptual decisions (e.g., Chang, Kanai, \& Seth, 2015; Doherty, Rao, Mesulam, \& Nobre, 2005; Sotiropoulos, Seitz, \& Seriès, 2011; Sterzer, Frith, \& Petrovic, 2008; Weiss, Simoncelli, \& Adelson, 2002; Wyart, Nobre, \& Summerfield, 2012; Chalk et al., 2010; Pinto et al., 2015). In the light of these successes, we have implemented a Bayesian model to explain our behavioral data.

To explain our behavioral data and capture the temporal dynamics, we implemented a recursive Bayesian updating scheme on a trial by trial basis. Critically, in our model the prior did not remain fixed in a single trial, instead it was updated at each iteration (Bitzer, Park, Blankenburg,

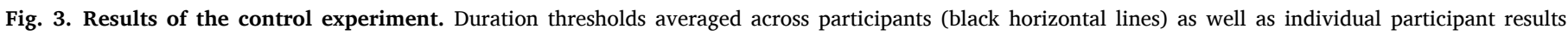

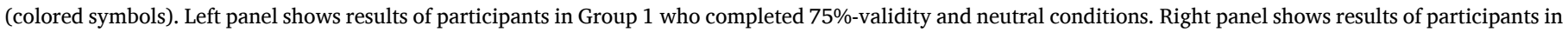
Group 2 who completed 50\%-validity and neutral conditions. 
\& Kiebel, 2014). Namely, in the first iteration for a trial, the prior was given to the model based on the cue and its validity. In subsequent iterations, however, the prior was updated with the posterior of the previous iteration. The posterior was computed by combining the prior and likelihood (based on the sensory input and a generative model) in each iteration. Fig. 4 outlines the steps of the algorithm. As can be seen in Fig. 4, the calculations were done separately for the observation on the left side and right side of the screen in each trial of the experiment. Below we explain the model in more detail.

We first defined feature values for the input (light gray boxes in Fig. 4)

$s= \begin{cases}s_{1}=-1 & \text { for a house image } \\ s_{2}=0 & \text { for a scrambled image } \\ s_{3}=1 & \text { for a face image }\end{cases}$

These would be the abstracted values received by the system if there were no noise. Next, we postulated that the abstracted observation extracted by the system, $x_{t}$, is drawn from a normal distribution

$x_{t} \sim N\left(s, \sigma^{2}\right)$.

During each trial we calculated $x_{t}$ at iteration $t$ based on the presented images on the corresponding sides. Next, we defined generative models for each decision alternative, $A_{i}: A_{1}$ for house, $A_{2}$ for scrambled, and $A_{3}$ for face-image. We calculated the likelihood of $x_{t}$ under each decision alternative as

$p\left(x_{t} \mid A_{i}\right)=\frac{1}{\widehat{\sigma} \sqrt{2 \pi}} \exp \left(-\frac{\left(x_{t}-s_{i}\right)^{2}}{2 \widehat{\sigma}^{2}}\right)$.

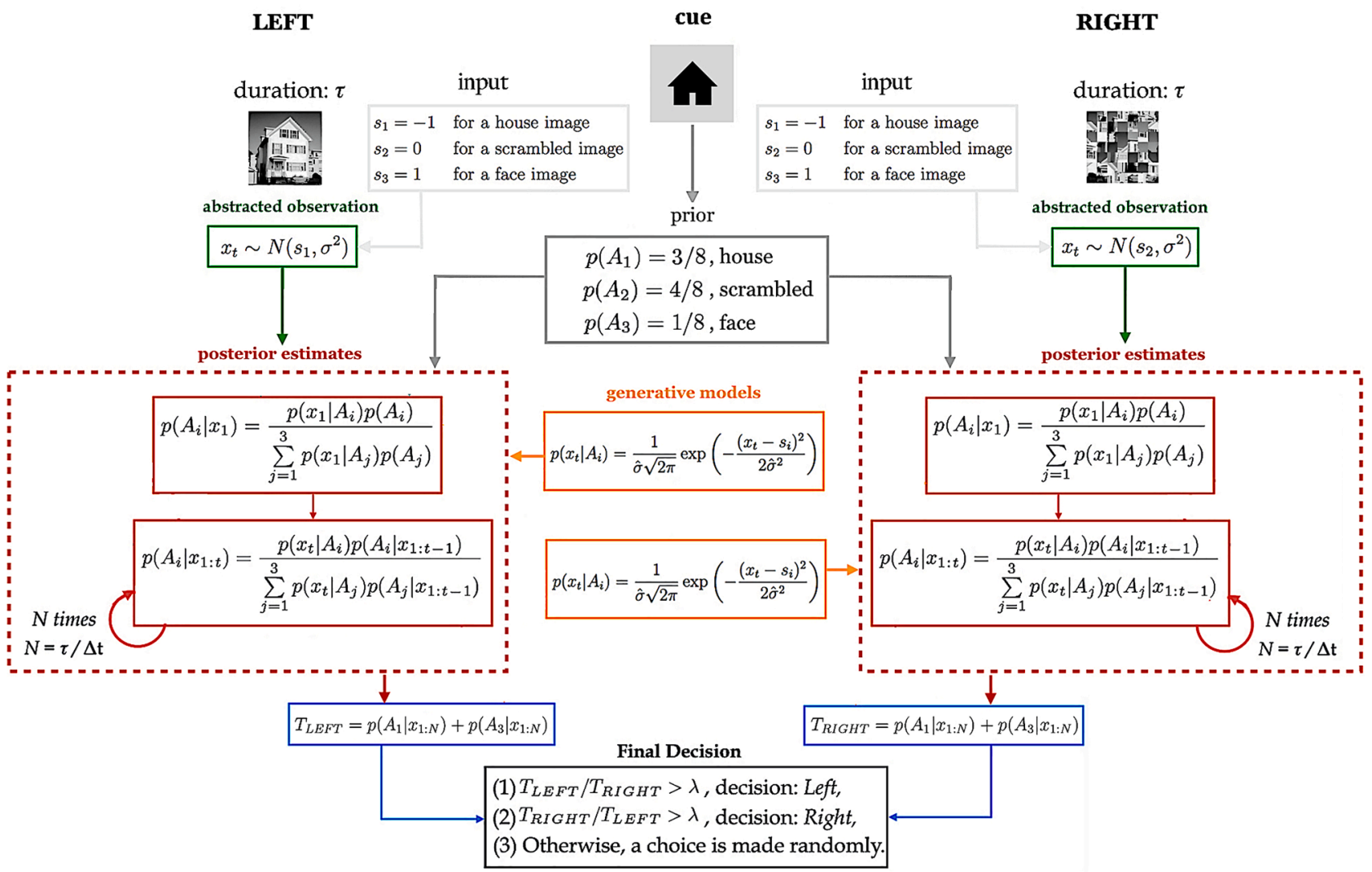

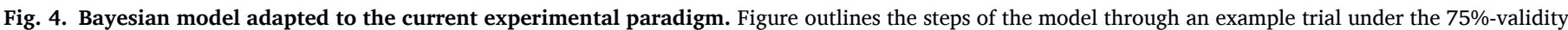

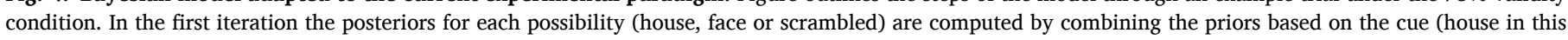

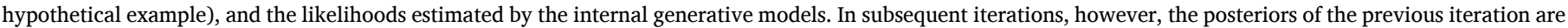

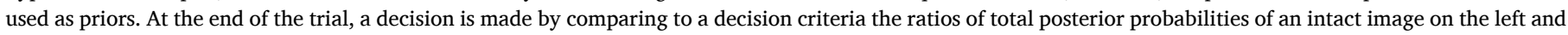

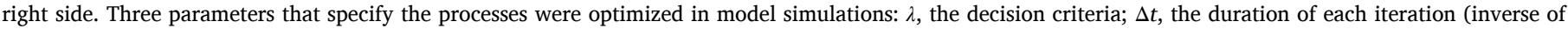
speed); $\widehat{\sigma}$, uncertainty of the generative model. See Table 1 for description of all the notations.
We defined the initial values of the priors as

$p\left(A_{i}\right)=c_{i}, \quad i=1,2,3$

where $c_{i}$ are defined based on the cue validity (i.e. $100 \%, 75 \%$ or $50 \%$ ), and the cue presented at that trial (i.e. face or house). For example in a trial under the $75 \%$-validity condition if the cue is a face then the priors are

$c_{1}=1 / 8, \quad c_{2}=4 / 8, \quad c_{3}=3 / 8$.

Next, we combined the likelihoods with the priors to compute posterior estimates for each decision alternative as follows

$p\left(A_{i} \mid x_{1}\right)=\frac{p\left(x_{1} \mid A_{i}\right) p\left(A_{i}\right)}{\sum_{j=1}^{3} p\left(x_{1} \mid A_{j}\right) p\left(A_{j}\right)}$.

Within a single trial posterior estimates are updated recursively over time ( $N$ times: number of iterations) until a decision is made by the model

$p\left(A_{i} \mid x_{1: t}\right)=\frac{p\left(x_{t} \mid A_{i}\right) p\left(A_{i} \mid x_{1: t-1}\right)}{\sum_{j=1}^{3} p\left(x_{t} \mid A_{j}\right) p\left(A_{j} \mid x_{1: t-1}\right)}$.

Note that, this amounts to using priors that are not fixed but updated in each iteration: posterior of the previous iteration becomes the prior for the next iteration. Number of iterations, $N$, in a single trial is

$N=\tau / \Delta t$

where $\tau$ represents the duration of presentation of the target images in determined by 
this particular trial, and $\Delta t$ defines how long each iteration lasts in the system. Next, we calculated the total probability of observing an intact image (face or house) for each side, $T_{L E F T}$ and $T_{R I G H T}$, as the sum of the final posterior of face-image and house-image (blue boxes in Fig. 4). At the last step, a final decision is made by the model using the criteria shown in black box in Fig. 4. Specifically, the ratio of $T_{L E F T}$ to $T_{R I G H T}$ is compared to the decision criteria, $\lambda$. This evaluation determines whether the model decides left or right. If this criteria cannot be met, then a decision is made randomly (see Table 1).

\subsubsection{Model simulations}

There were three free parameters that governed the computations: $\lambda$ (a decision criteria), $\Delta t$ (how long each iteration lasts; inverse of processing speed), and $\widehat{\sigma}$ (the internal uncertainty of the generative model, Eq. (3)). In a single trial, the ratio of the duration of that trial, $\tau$, and $\Delta t$ determine the number of iterations. Using the optimized parameter values (that minimize the error between the model's prediction and the real data) we ran 1000 simulations of the model to ensure the stability of the model's predictions (Ritter, Schoelles, Quigley, \& Klein, 2011) for each participant's data. We generated separate models for 100\%-, 75\%-, $50 \%$-validity conditions, and the model simulations were compared to the data of these validity conditions for each participant. Note that there was only a single difference between the models of different validity conditions, and it was the initial values of the priors (See gray box in Fig. 4). The neutral condition was not included in the simulations, because there was no explicit (informative) cue in the neutral condition, which made it inherently different than other conditions.

\subsubsection{Model comparison}

To test whether the underlying parameters of the system are altered in different conditions and trial types, we compared restricted and unrestricted models (Alternative 1 ). In the restricted model a single set of parameters (3 parameters: $\lambda, \Delta t$, and $\widehat{\sigma}$ ) was optimized for all validity conditions and trial types per participant. In the unrestricted (free) model 5 sets of parameters, one for every trial type (congruent, incongruent), and validity condition $(100,75,50 \%)$ were optimized per participant (15 parameters in total). If the internal parameters of the system do not change, we would expect no statistically significant improvement in the fits under the free model. Conversely, if the fits get better under the free model, we would conclude that the internal parameters of the system vary depending on the validity and trial type. Fig. $5 a$ shows Bayesian model simulation results for a single participant (see Supplementary Material for simulation results of all participants along with psychometric functions fits). It is first worth noting that the model can successfully capture the pattern observed in the empirical data: just as the human participants, the model needs more time to complete the sensory process for incongruent trials in the $75 \%$ validity condition (the fits shift

Table 1

Notations for Bayesian model.

\begin{tabular}{|c|c|}
\hline Symbol & Description \\
\hline$s$ & $\begin{array}{l}\text { Feature (category) value that would be extracted by the system under a } \\
\text { noise-free observation. 1: House; 0: Scrambled; }-1 \text { : Face }\end{array}$ \\
\hline$x_{t}$ & $\begin{array}{l}\text { Noisy sensory input at iteration } t \text {, drawn from a normal distribution with } \\
\text { mean } s\end{array}$ \\
\hline$A_{i}$ & Decision alternatives (house, face or scrambled) \\
\hline$p\left(A_{i}\right)$ & Prior probabilities of the alternatives \\
\hline$p\left(x_{t} \mid A_{i}\right)$ & Likelihood of the observation, $x_{t}$, under the alternative $A_{i}$ \\
\hline$p\left(A_{i} \mid x_{t}\right)$ & Posterior probability of alternative $A_{i}$ given the observation, $x_{t}$ \\
\hline$\tau$ & Presentation duration of the stimulus in the trial \\
\hline$\lambda$ & Decision criteria (free parameter) \\
\hline$\Delta t$ & $\begin{array}{l}\text { How long each iteration lasts in the system (inverse of speed, free } \\
\text { parameter) }\end{array}$ \\
\hline$\widehat{\sigma}$ & Internal uncertainty of the generative models (free parameter) \\
\hline$N$ & Number of iterations performed in the trial, $\tau / \Delta t$ \\
\hline
\end{tabular}

to the right for incongruent trials), whereas no such difference is observed in the 50\%-validity condition.

Once the optimized parameters were computed, we applied chisquare nested hypothesis tests at the individual participant level to find whether the unrestricted model could explain the behavioral data better than the restricted model. Under the null hypothesis, twice the difference between the log-likelihoods of the two models has an approximate chi-square distribution, with degrees of freedom equal to 12 , which is the difference in the number of free parameters between the two models at the individual participant level. Thus we reject the null hypothesis if

$2 \times\left(\log L_{1}-\log L_{0}\right) \geqslant \chi_{12}^{2}$,

where the likelihoods $L_{0}$ and $L_{1}$ are calculated for the restricted and unrestricted model respectively

$L .=\prod_{i=1}^{n} P\left(Y_{i} \mid B_{\text {model }}\right)$

where $n$ is equal to the total number of trials in each experimental condition, $Y_{i}$ corresponds to the participant's response, and $B_{\text {model }}$ is the model's prediction at the $i$-th trial.

The results of the likelihood-ratio tests showed that the two models are not different from each other in any participant $(p>0.05)$. This suggests that the internal parameters $(\lambda, \Delta t, \widehat{\sigma})$ do not change with expectation and cue validity. This refutes the first alternative we proposed earlier to explain the human data.

Recall that according to the other alternative the system simply needs to further process the visual input to converge on a decision when the stimulus category is unexpected. Thus, this alternative requires that the number of iterations should be greater in incongruent trials compared to congruent trials. To test this, we calculated the number of iterations performed by the (restricted) model in congruent and incongruent trials in all validity conditions. Fig. $5 b$ shows the results. We performed a 2 (trial type: congruent, incongruent) $\times 2$ (validity: $75 \%$, $50 \%$ ) repeated measures ANOVA to investigate the effect of expectation and validity on number of iterations. The main effect of trial type was significant $(F(1,7)=13.987, p=0.007)$. However, the main effect of validity and interaction were not significant $(F(1,7)=0.348, p=0.574$; $F(1,7)=0.669, p=0.440$ ). Next, we examined whether the number of iterations differ based on expectation in each validity condition. We found a significant difference between the congruent and incongruent trials in the $75 \%$ validity condition $(t(7)=3.467, p=0.010)$. There was no difference between the trial types in the $50 \%$ validity condition $t(7)=$ $1.950, p=0.092$. We also found no difference between the $100 \%$ and the congruent trials of both $75 \%$ and $50 \%$ validity conditions $(t(7)=1.085$, $p=0.314 ; t(7)=1.948, p=0.092$ ). Overall the Bayesian model results agree remarkably well with the behavioral data, and suggest that the longer thresholds for the incongruent trials can be explained simply by further processing required when expectations are not met.

\section{Discussion}

In this study we found that temporal detection thresholds in an individuation task are longer when expectations are not met. Using the recursive Bayesian model with dynamic priors, we showed that this empirical effect can be explained simply by further processes required in the system to converge on a decision when expectations are not met, and no internal parameters need to be altered. Our experimental procedures and model offer a novel framework that can easily be extended to study the effects of expectation on other visual features, and other sensory processes. 

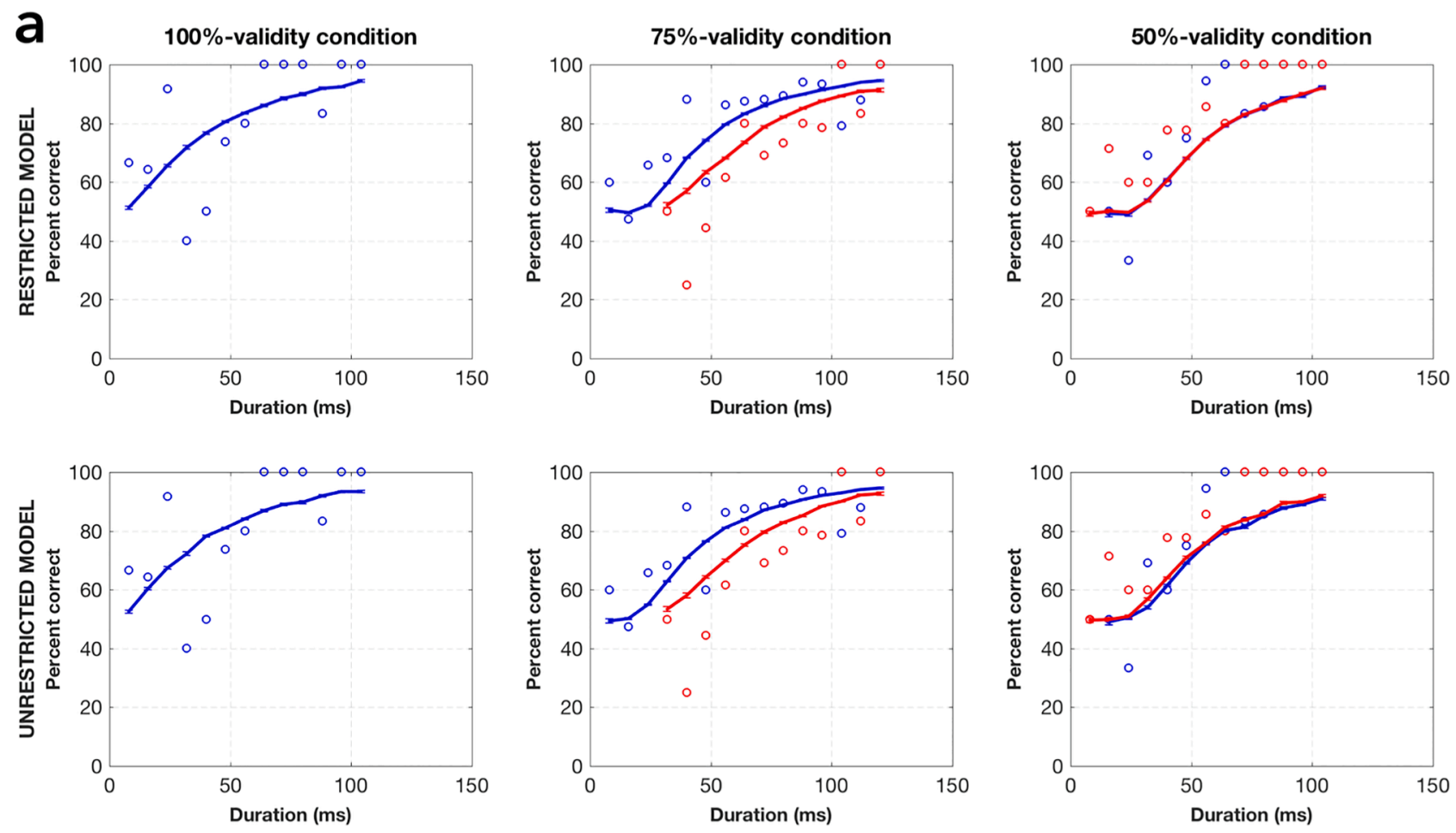

$\begin{array}{ll}\circ \text { congruent data } & - \text { model simulation - congruent } \\ \circ \text { incongruent data } & - \text { model simulation - incongruent }\end{array}$
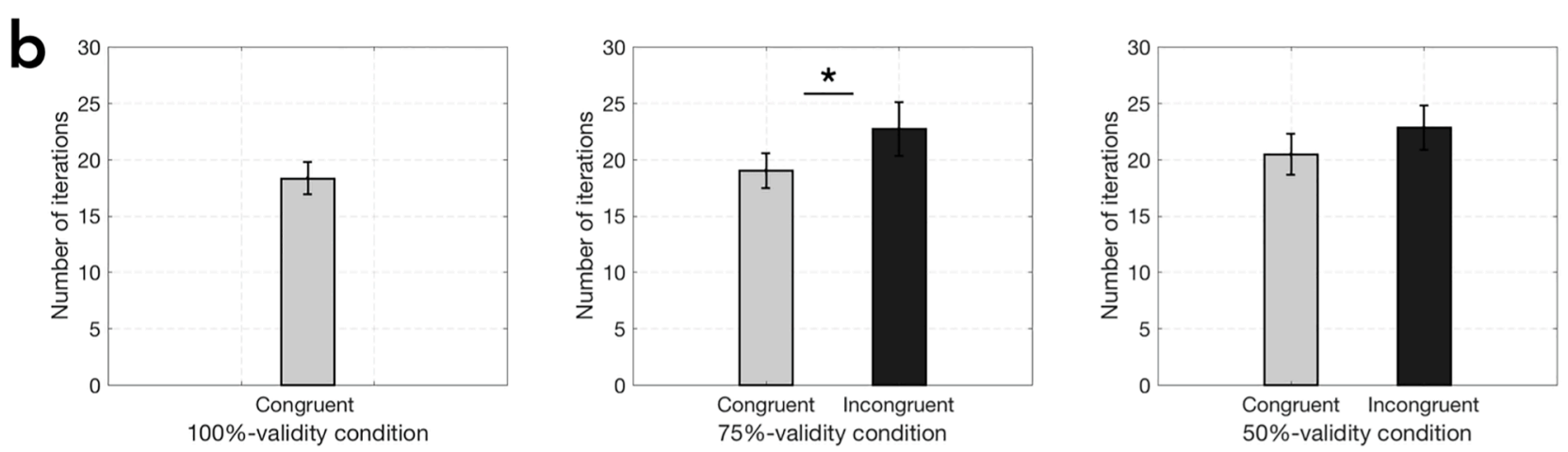

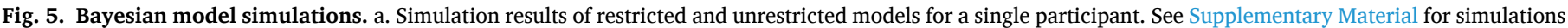

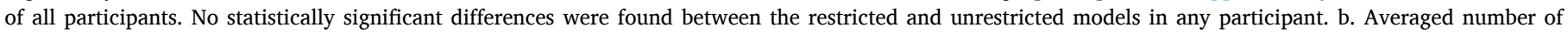

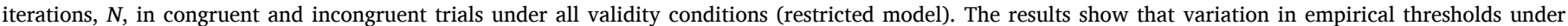

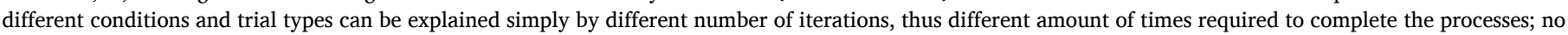
internal parameters need to be altered. Error bars are twice the standard error.

\subsection{Detecting an unexpected stimulus requires longer time}

In the behavioral experiment, we found no threshold differences between the $100 \%$ validity and neutral conditions, and between the $100 \%$ and congruent trials of both $75 \%$ and $50 \%$ validity conditions. We were surprised with these results, because unlike the results reported in perceptual decision literature, they show that expectation does not facilitate early sensory processes. On the other hand the thresholds were longer for the incongruent trials of the $75 \%$ validity condition compared to both $100 \%$ validity condition and the congruent trials of the $75 \%$ validity condition. Together, these results show that fulfilled expectations do not reduce thresholds, instead thresholds increase when expectations are not met.

Expectations (also named as prediction, priming or congruency in different experimental paradigms) are consistently shown to affect perceptual processes (e.g. lead to quicker decisions, or increased precision, e.g. Jabar \& Anderson, 2017, to the expected stimulus). Specifically, in several studies expectations are typically argued to facilitate perceptual decisions. Yet, we show that expectations do not facilitate sensory processes, instead unmet expectations slow them down. How can we reconcile this disagreement? The disagreement could be because of distinct mechanisms involved in perceptual decisions and sensory processes. Here we have tested the early sensory processes by measuring temporal thresholds, whereas in perceptual decision studies usually accuracies and response times (RTs) are measured. RTs reflect the time required by a combination of early sensory, cognitive, decision-making and motor processes. Thus, it could well be that perceptual decisions are facilitated for the expected stimuli even though low-level processes are not. On the other hand, we were able to uncover the exact nature of the difference between expected and unexpected stimuli only by 
systematically varying the validity $(100,75,50 \%$ and neutral). Often in perceptual decision studies this subtlety is overlooked, and simply expected and unexpected stimuli are compared under a fixed validity condition, which may lead to a somewhat misleading conclusion. Indeed, in a very similar individuation task as the one used here, comparing RTs in congruent, incongruent, and neutral trials, De Loof et al. (2016) found that perceptual decisions with unexpected stimuli required longer times than both expected and neutral stimuli, and there was no difference between expected and neutral stimuli (De Loof et al., 2016, Fig. 3.). Those results are in agreement with our results. Therefore the disagreement between our results and results of many other perceptual decision studies may be ostensible. Future studies are needed to find out whether the thresholds of valid expectations would change under different experimental conditions or with different class of stimuli.

\subsection{Unexpected stimulus delays the sensory processes}

How can our behavioral findings be explained? To investigate this, we used a recursive Bayesian model, where the priors were not fixed but updated as the processes unfold in a trial. Model simulations showed that expectation and its validity do not alter the underlying parameters of the system (e.g. decision criteria, and processing speed). The simulations showed, however, that the patterns of behavioral results could be explained simply by more number of iterations, thus a longer time, required by the system to complete the sensory processes. Conceptually, in our model the events in an incongruent trial unfold as follows: when an unexpected stimulus is presented, the sensory input does not agree with the initial prior information. Because of this conflict, the posterior probabilities cannot reach a sufficiently strong level to favor a left or a right decision. As more iterations are performed, the priors are updated, and they become more inline with the sensory input. Therefore sufficient amount of evidence can be acquired only after a longer process for the unexpected stimulus compared to the expected stimulus.

In a study that we discussed earlier, using a similar experimental design De Loof et al. (2016) studied perceptual decisions by measuring RTs. In that study, the authors used drift-diffusion model (DDM) (Ratcliff, 1978) to model the empirical results, and found that the unexpected stimuli led to increased boundary separation, which is defined as the internal threshold that is required to reach a decision (De Loof et al., 2016; see also Bang \& Rahnev, 2017). (Note that DDM is shown to be consistent with recursive Bayesian models, Bitzer et al., 2014). This result appears at odds with our findings. The disagreement, once again, could be a result of differences between sensory processes and perceptual decisions. Alternatively, the disagreement could be because of the differences in experimental design and analysis procedures. Even though DDM is a well-studied and highly useful model to understand the underlying processes in perceptual decisions, it does not capture the temporal dynamics throughout a single trial (Huk, Bonnen, \& He, 2018). Our experimental procedure, which gives us a temporally pseudocontinuous set of data, and the models we used, allowed us to uncover the dynamics of the events in a single trial. Thus reconciling the differences may require more careful investigation of the dynamics of behavior during perceptual decisions.

Our findings are in line with several studies (Vincent, 2011; Gekas, Seitz, \& Seriès, 2015) that investigated whether humans can integrate prior knowledge and sensory input in a Bayes optimal fashion using similar localization tasks. For instance, Gekas et al. (2015) showed that people can rapidly learn stimulus distribution throughout a task without an overt cue, and can (near-optimally) integrate implicitly learned priors with the sensory evidence while this process is also affected by recent history of trials in the experiment. Also, by varying validity of prior knowledge Vincent (2011) showed that the pattern observed in human behavior (across validity conditions) can be very closely captured within Bayesian framework. It should also be noted that the experimental paradigms used in that study might lead observed findings to be a result of the combined effects of expectation and attention. In our study, however, the cue provided task-irrelevant prior information. Specifically, one did not have to distinguish the category of the target stimulus, which was the expected feature, to accomplish the (localization) task. We made this conscious choice in order to eliminate or minimize the effect of attention on our results (see e.g. Summerfield \& De Lange, 2014 for the distinction between attention and expectation).

\subsection{Possible neural mechanisms underpinning the delay in sensory processes}

We showed that a recursive Bayesian model is successful to explain the behavioral results, but how can such a model be implemented by the biological nervous system? Firstly, it has been shown that population responses of neurons can indeed implement a Bayesian decision making algorithm (Ma, Beck, Latham, \& Pouget, 2006; van Bergen, Ma, Pratte, \& Jehee, 2015; Walker, Cotton, Ma, \& Tolias, 2020). Secondly, a number of mechanistic neuronal models have been put forward to approximate Bayesian inference in brain (e.g., Mumford, 1992, Rao \& Ballard, 1999, Friston, 2005, Heeger, 2017). Among those, predictive coding models hypothesize that higher level brain areas generate a model of the world that is relayed to lower levels. In the lower levels, sensory input and those predictions (after a transformation) are compared, and any residual error is signaled back to higher levels to correct and refine the models of the visual world. Therefore implementation of predictive coding models in brain require an interplay between representation units and at least one type of error units. The process continues until an equilibrium is reached (Clark, 2013; Keller \& Mrsic-flogel, 2018). Such an updating mechanism would be perfectly consistent with the recursive Bayesian model we propose here, and lead to a longer duration of neuronal processing and thus potentially explain the higher temporal thresholds we observed in the current experiment.

Of course predictive coding models are not the only possible neuronal models with which Bayesian inference can be implemented in brain. For example, a recently proposed model by Heeger (2017) posits that the activity of neural units can be thought as a weighted response to bottom-up sensory input and top-down prior drive. Here, the model does not require explicitly comparing high-level predictions and low-level sensory inputs, nor specifying distinct subpopulations of error and representation units. Our preliminary simulations using such a model showed that when expectations are not met, higher number of neural units may become active, and resolving the conflict between sensory input and the priors may require additional and longer computations. Note that this is exactly what we see in the Bayesian model, as well. Such a parsimonious scenario could naturally cause both a delay in human behavior and a higher brain response. Also note that the delay found in unmet expectations in the current study is suggested to be in early sensory processing, because we measured duration thresholds, a method that provides us to isolate sensory processes from higher level decisions. On the other hand, it should also be noted that the observed delay does not necessarily happen in early visual areas, such as V1, but may be observed in later stages of early sensory processing, including FFA and PPA. Future investigations, using neuroimaging or other recording techniques as well as rigorous modeling efforts, are needed to unravel which of the possible neural mechanisms can better explain the effect of expectations.

\section{Conclusion}

In conclusion, we demonstrated that expectations affect low-level sensory processes. Specifically, sensory processes are delayed when relatively strong expectations are not met. To explain these empirical findings, we implemented a recursive Bayesian model. The model simulations showed that the delay in sensory processes is the result of longer computations required in case of violated expectations. We found no evidence that internal parameters of the system are altered. We contend 
that the effect of expectations on sensory processes can be revealed with a parsimonious computational model we introduce here, which has the potential to be expanded and used for new and novel studies.

\section{Data and code availability}

Data and code used in the study is available at https://doi.org/ 10.6084/m9.figshare.13326473.v1 (Urgen \& Boyaci, 2020).

\section{Author contributions}

$\mathrm{HB}$ and BMU conceived the original study. BMU and HB designed the behavioral experiment, and BMU implemented the model. BMU collected and analyzed all data with support from HB.

\section{CRediT authorship contribution statement}

Buse M. Urgen: Conceptualization, Methodology, Software, Formal analysis, Investigation, Writing - original draft, Writing - review \& editing, Visualization. Huseyin Boyaci: Conceptualization, Methodology, Writing - review \& editing, Supervision, Funding acquisition.

\section{Declaration of Competing Interest}

The authors declare that they have no known competing financial interests or personal relationships that could have appeared to influence the work reported in this paper.

\section{Acknowledgements}

This work was funded by a grant of the Turkish National Scientific and Technological Council (TÜBITAK 217K163) awarded to HB. We thank Katja Doerschner for her valuable comments on an earlier version of the manuscript.

\section{Appendix A. Supplementary data}

Supplementary data associated with this article can be found, in the online version, at https://doi.org/10.1016/j.visres.2020.12.004.

\section{References}

Aitken, F., Turner, G., \& Kok, P. (2020). Prior expectations of motion direction modulate early sensory processing. Journal of Neuroscience, 40(33), 6389-6397.

Bang, J. W., \& Rahnev, D. (2017). Stimulus expectation alters decision criterion but not sensory signal in perceptual decision making. Scientific Reports, 7(1), 1-12.

Bar, M. (2004). Visual objects in context. Nature Reviews Neuroscience, 5(8), 617.

Bitzer, S., Park, H., Blankenburg, F., \& Kiebel, S. J. (2014). Perceptual decision making: drift-diffusion model is equivalent to a bayesian model. Frontiers in Human Neuroscience, 8, 102.

Brainard, D. H. (1997). The psychophysics toolbox. Spatial Vision, 10, 433-436.

Chalk, M., Seitz, A. R., \& Seriès, P. (2010). Rapidly learned stimulus expectations alter perception of motion. Journal of Vision, 10(8), 2-2.

Chang, A. Y.-C., Kanai, R., \& Seth, A. K. (2015). Cross-modal prediction changes the timing of conscious access during the motion-induced blindness. Consciousness and Cognition, 31, 139-147.

Clark, A. (2013). Whatever next? Predictive brains, situated agents, and the future of cognitive science. Behavioral and Brain Sciences, 36(3), 181-204.

de Lange, F. P., Heilbron, M., \& Kok, P. (2018). How do expectations shape perception? Trends in Cognitive Sciences.

De Loof, E., Van Opstal, F., \& Verguts, T. (2016). Predictive information speeds up visual awareness in an individuation task by modulating threshold setting, not processing efficiency. Vision Research, 121, 104-112.

Doherty, J. R., Rao, A., Mesulam, M. M., \& Nobre, A. C. (2005). Synergistic effect of combined temporal and spatial expectations on visual attention. Journal of Neuroscience, 25(36), 8259-8266.
Friston, K. (2005). A theory of cortical responses. Philosophical Transactions of the Royal Society of London B: Biological Sciences, 360(1456), 815-836.

Gekas, N., Seitz, A. R., \& Seriès, P. (2015). Expectations developed over multiple timescales facilitate visual search performance. Journal of Vision, 15(9), 10-10.

Heeger, D. J. (2017). Theory of cortical function. Proceedings of the National Academy of Sciences, 114(8), 1773-1782.

Helmholtz, H. v. (1866). Concerning the perceptions in general. Treatise on Physiological Optics.

Huk, A., Bonnen, K., \& He, B. J. (2018). Beyond trial-based paradigms: Continuous behavior, ongoing neural activity, and natural stimuli. Journal of Neuroscience, 38 (35), 7551-7558.

Jabar, S. B., \& Anderson, B. (2017). Not all probabilities are equivalent: Evidence from orientation versus spatial probability learning. Journal of Experimental Psychology: Human Perception and Performance, 43(5), 853.

Keller, G. B., \& Mrsic-flogel, T. D. (2018). Predictive processing: A canonical cortical computation. Neuron, 100(2), 424-435.

Kersten, D., Mamassian, P., \& Yuille, A. (2004). Object perception as bayesian inference. Annual Review of Psychology, 55, 271-304.

Kersten, D., \& Yuille, A. (2003). Bayesian models of object perception. Current Opinion in Neurobiology, 13(2), 150-158.

Kingdom, F. A., \& Prins, N. (2010). Psychophysics: A practical introduction. Academic Press London.

Knill, D. C., \& Pouget, A. (2004). The bayesian brain: The role of uncertainty in neural coding and computation. Trends in Neurosciences, 27(12), 712-719.

Knill, D. C., \& Richards, W. (1996). Perception as Bayesian Inference. Cambridge University Press.

Kok, P., Jehee, J. F., \& De Lange, F. P. (2012). Less is more: Expectation sharpens representations in the primary visual cortex. Neuron, 75(2), 265-270.

Ma, W. J., Beck, J. M., Latham, P. E., \& Pouget, A. (2006). Bayesian inference with probabilistic population codes. Nature Neuroscience, 9(11), 1432-1438.

Maloney, L. T., \& Mamassian, P. (2009). Bayesian decision theory as a model of human visual perception: Testing bayesian transfer. Visual Neuroscience, 26(1), 147-155.

Mamassian, P., Landy, M., \& Maloney, L. T. (2002). Bayesian modelling of visual perception. Probabilistic Models of the Brain, 13-36.

Minear, M., \& Park, D. C. (2004). A lifespan database of adult facial stimuli. Behavior Research Methods, Instruments, \& Computers, 36(4), 630-633.

Mumford, D. (1992). On the computational architecture of the neocortex. Biological Cybernetics, 66(3), 241-251.

Pinto, Y., Gaal, S. van, Lange, F. P. de, Lamme, V. A., \& Seth, A. K. (2015). Expectations accelerate entry of visual stimuli into awareness. Journal of Vision, 15(8), 13-13.

Rao, R. P., \& Ballard, D. H. (1999). Predictive coding in the visual cortex: A functional interpretation of some extra-classical receptive-field effects. Nature Neuroscience, 2 (1), 79.

Ratcliff, R. (1978). A theory of memory retrieval. Psychological Review, 85(2), 59.

Ritter, F. E., Schoelles, M. J., Quigley, K. S., \& Klein, L. C. (2011). Determining the number of simulation runs: Treating simulations as theories by not sampling their behavior. In Human-in-the-loop simulations (pp. 97-116). Springer.

Rungratsameetaweemana, N., Itthipuripat, S., Salazar, A., \& Serences, J. T. (2018). Expectations do not alter early sensory processing during perceptual decisionmaking. The Journal of Neuroscience, 38(24), 5632-5648.

Sotiropoulos, G., Seitz, A. R., \& Seriès, P. (2011). Changing expectations about speed alters perceived motion direction. Current Biology, 21(21), R883-R884.

Sterzer, P., Frith, C., \& Petrovic, P. (2008). Believing is seeing: Expectations alter visual awareness. Current Biology, 18(16), R697-R698.

Summerfield, C., \& De Lange, F. P. (2014). Expectation in perceptual decision making: Neural and computational mechanisms. Nature Reviews Neuroscience, 15(11), 745.

Urgen, B. M., \& Boyaci, H. (2020). Unmet expectations delay sensory processes.

van Bergen, R. S., Ma, W. J., Pratte, M. S., \& Jehee, J. F. (2015). Sensory uncertainty decoded from visual cortex predicts behavior. Nature Neuroscience, 18(12), 1728-1730.

Vincent, B. (2011). Covert visual search: Prior beliefs are optimally combined with sensory evidence. Journal of Vision, 11(13), 25-25.

Walker, E. Y., Cotton, R. J., Ma, W. J., \& Tolias, A. S. (2020). A neural basis of probabilistic computation in visual cortex. Nature Neuroscience, 23(1), 122-129.

Weiss, Y., Simoncelli, E. P., \& Adelson, E. H. (2002). Motion illusions as optimal percepts. Nature Neuroscience, 5(6), 598.

Willenbockel, V., Sadr, J., Fiset, D., Horne, G. O., Gosselin, F., \& Tanaka, J. W. (2010). Controlling low-level image properties: The shine toolbox. Behavior Research Methods, 42(3), 671-684.

Wyart, V., Nobre, A. C., \& Summerfield, C. (2012). Dissociable prior influences of signal probability and relevance on visual contrast sensitivity. Proceedings of the National Academy of Sciences, 109(9), 3593-3598.

Xiao, J., Hays, J., Ehinger, K. A., Oliva, A., \& Torralba, A. (2010). Sun database: Largescale scene recognition from abbey to zoo. In 2010 IEEE conference on Computer vision and pattern recognition (cvpr) (pp. 3485-3492).

Yuille, A., \& Kersten, D. (2006). Vision as bayesian inference: Analysis by synthesis? Trends in Cognitive Sciences, 10(7), 301-308. 